\title{
Malondialdehyde in early phase of acute pancreatitis
}

\author{
Vicent Hernández ${ }^{1}$, María Miranda ${ }^{2}$, Isabel Pascual ${ }^{1}$, Vicente Sánchiz ${ }^{1}$, Pedro Almela ${ }^{1}$, Ramón Añón ${ }^{1}$, \\ Esperanza Cuadrado ${ }^{1}$, María Isabel Sanz ${ }^{1}$, Miguel Mínguez ${ }^{1}$, Francisco Mora ${ }^{1}$, Francisco Javier Romero ${ }^{2}$ \\ and Adolfo Benages ${ }^{1}$
}

${ }^{I}$ Department of Gastroenterology. Hospital Clínico Universitario de Valencia. Universitat de València, Spain. ${ }^{2}$ Department of Phisiology, Pharmacology and Toxicology. Universidad Cardenal Herrera-CEU. Moncada. Valencia, Spain

\begin{abstract}
Aims: to assess oxidative stress in acute pancreatitis, its evolution over time and its relationship with the severity of the disease.

Methods: during a two-year period, patients with acute pancreatitis with less than 24 hours of pain were evaluated. Serum was obtained the first, second and fourth day from admittance, if complications were detected, and after recovery. Malondialdehyde was determined by high performance liquid chromatography. Twenty healthy volunteers constituted the control group. Malondialdehyde between groups was compared with Mann-Whitney and KruskalWallis tests; malondialdehyde evolution was studied with Wilcoxon test.

Results: one hundred and sixty-nine patients were included (91 women, median age 67 years, range 20-95); 33 suffered a severe episode. Malondialdehyde decreased from first to fourth day $(0.600$ vs. 0.451 vs. $0.343 \mathrm{M}$, respectively, $\mathrm{p}<0.05)$. When complications were detected, malondialdehyde level was similar to that of first and second day (0.473 M, p >0.05). In severe attacks malondialdehyde was higher than in control group at day 2 (severe: 0.514 ; mild: 0.440; control: $0.347 \mathrm{M}, \mathrm{p}<0.05$ severe us. control).

Conclusions: an early oxidative stress is observed in acute pancreatitis. In severe attacks, oxidative stress remains high longer than in mild episodes. The onset of complications is associated with high malondialdehyde concentration.
\end{abstract}

Key words: Oxidative stress. Malondialdehyde. Acute pancreatitis. Complications

Hernández V, Miranda M, Pascual I, Sánchiz V, Almela P, Añón $R$, Cuadrado E, Sanz MI, Mínguez M, Mora F, Romero FJ, Benages A. Malondialdehyde in early phase of acute pancreatitis. Rev Esp Enferm Dig 2011; 103: 563-569.

Received: 18-04-11.

Accepted: 25-05-11.

Correspondence: Adolfo Benages. Department of Gastroenterology. Hospital Clínico Universitario de Valencia. Avda. Blasco Ibañez, 17. 46010 Valencia, Spain.

e-mail: adolfo.benages@uv.es

\section{INTRODUCTION}

Acute pancreatitis (AP) is an acute inflammatory process of the pancreatic gland, with variable involvement of other regional tissues or remote organ systems. Severe attacks are defined by the presence of multisystemic organ failure (MOF) or local complications (necrosis, abscess or pseudocyst) (1). Pancreatitis-related mortality can be regarded as "early" or "late". "Late mortality" is usually the consequence of sepsis secondary to infectious complications; the improvement of supportive care, the use of enteral feeding and the restriction of necrosectomy to infected necrosis have possibly led to a fall in sepsis-related deaths (2). However, "early mortality" remains a challenge in clinical practice. Early events in the course of AP can be explained by the inflammatory cascade activation with the development of systemic inflammatory response syndrome (SIRS) and MOF. Some authors have proposed the existence of a window period in early phases of the disease, in which a treatment directed to the control of inflammatory activation could improve its outcome (3).

Since the first description of the involvement of oxygen free radicals in the pathophysiology of AP made by Sanfey et al. (4), mounting experimental and clinical evidence of the role of oxidative stress (OS) in AP has been accumulated. Clinical studies have shown that OS is higher in patients with AP than in healthy volunteers or patients with acute abdominal processes other than AP (5-15), and in patients with severe AP (SAP) it is higher than in those with mild AP (MAP) $(8-14,16,17)$.

OS can be assessed by measuring the level of antioxidants or the concentration of substances derived from the action of oxygen free radicals on biological molecules, such as malondialdehyde (MDA). Measurement of MDA is considered an effective marker of OS in a biological sample (18) and, together with the analysis of thiobarbituric acid reactive substances (TBARS), is the usual method to assess OS in human AP $(7,8,10,11,14,15,17,19-21)$. 
Studies on the evolution of MDA levels in the early phase of the disease are controversial: some authors have found a different evolutive pattern between MAP and $\operatorname{SAP}(8,14)$, while others have not appreciated such differences $(11,15,17,21)$.

If the hypothesis of the window period in the early phase of AP is accepted, taking into account the important role that oxygen free radicals play in the activation of the inflammatory cascade, a knowledge of the evolution of OS in the early phase of AP and its relationship with the development of complications could help in the management of the disease from a pathophysiological perspective.

Therefore, the aim of our study was to assess the magnitude of OS in AP (analyzing MDA levels), its evolution over time and its relationship with the severity of the disease.

\section{PATIENTS AND METHODS}

From the $1^{\text {st }}$ December 2001 to $30^{\text {th }}$ November 2003, a prospective observational study was conducted on patients admitted with the diagnosis of AP, who attended the emergency area of the hospital within the first 24 hours from the beginning of abdominal pain. Patients were excluded if they referred pain for more than 24 hours at the moment of exploration in the emergency area of the hospital or suffered from chronic pancreatitis, pancreatic cancer or diseases in which OS plays an important pathogenic role (inflammatory bowel disease, chronic renal failure, hepatic cirrhosis, recently diagnosed neoplasia).

Patients were classified into MAP and SAP according to the Atlanta criteria. Blood samples were obtained for the usual analytical study and for the determination of MDA, at the first and second day of stay in the hospital (named 24 and 48 hours), at the fourth day (named 96 hours, only for MDA determination), when any complication was detected and at the moment when the patient was considered recovered from the episode, at least one month after the attack (named recovery). Serum samples for the determination of MDA were immediately frozen at $-80^{\circ} \mathrm{C}$ until the moment of MDA determination.

Serum MDA concentration (MDAc) was measured according to a modification of the method of Richard (22), developed by Romero et al. (23). After reaction with thiobarbituric acid, the adduct MDA-thiobarbituric acid was measured by high performance liquid chromatography (HPLC).

To obtain the normal values of MDAc, a control group (CG) was constituted with 20 healthy volunteers (10 men/10 women; median age, 38 years; range 30-55).

Variables were expressed as median (interquartilic range, IQR), apart from analytical parameters shown as mean (95\% confidence interval, CI95\%). Student-t test was used to compare analytical parameters between MAP and SAP. Non-parametric tests were used to compare MDAc between the different groups: Kruskal-Wallis and Mann-Whitney tests were carried out to compare MDAc between CG, MAP and SAP; the Wilcoxon test for paired data was performed to evaluate the evolution of MDAc over time. The relationship between analytical parameters and MDAc was assessed using Spearman's coefficient $\left(\mathrm{r}_{\mathrm{s}}\right)$. Statistical significance was assumed if $p<0.05$. To assess the usefulness of MDA levels as prognostic factor of severity, ROC curves were drawn and the best cut-off value was used to calculate the sensitivity, specificity, positive predicting value and negative predicting value. These parameters were compared with those of Ranson and Glasgow criteria and the C-reactive protein peak within the first 48 hours, which are widely accepted prognostic criteria. Variables shown to have differences between MAP and SAP $(p<0.05)$ were included in a logistic regression model to estimate their importance as risk factor of severity, calculating the odds ratio with 95\% confidence interval. The statistical analysis was performed using the SPSS program version 12.0 (SPSS Inc., Chicago, Illinois, USA).

The study was reviewed and approved by the Ethics Committee of the University Clinical Hospital of Valencia, Spain, and was conducted following the Ethical Principles of the Helsinki Declaration.

\section{RESULTS}

In the study period, 259 patients were admitted due to an AP attack. Ninety patients were excluded: 37 suffered from abdominal pain for more than 24 hours, 16 suffered from chronic pancreatitis or this diagnostic was established as a consequence of the acute attack, 1 patient was diagnosed of pancreatic neoplasia, 18 suffered from diseases related to OS, and in 18 patients blood samples could not be collected in the acute phase of the disease. In 120 patients blood samples were collected both in the acute phase and at recovery, while in 49 patients blood samples could be collected only in the acute phase of the disease. As we aimed to assess the OS in the early phase of AP, these 49 patients were included in the study.

Therefore, the study group was formed by 169 patients (78 men/91 women), median age 67 years, range 20-95. In 113 patients the AP was due to gallstones, 21 suffered an alcoholic AP, other causes were found in 3 patients, and in 32 the cause could not be established. According to the Atlanta criteria, 33 patients (19.5\%) presented a severe episode and 136 patients a mild attack. Five patients (3\%) needed intensive treatment in the Intensive Care Unit. Necrosectomy was performed in one patient because of pancreatic infection. Two patients died as a result of the AP episode ( $1.2 \%$ of the patients, $6.1 \%$ of SAP episodes).

\section{MDAc in patients with AP}

Compared to the CG, patients with AP showed significantly higher MDAc at 24 and 48 hours from admit- 
Table I. MDAC $(\mu \mathrm{M})$ at each time point and evolution in patients with Al

\begin{tabular}{|c|c|c|c|c|c|c|}
\hline & & $\begin{array}{l}24 \text { hours } \\
(n=169)\end{array}$ & $\begin{array}{l}48 \text { hours } \\
(n=169)\end{array}$ & $\begin{array}{l}96 \text { hours } \\
(n=169)\end{array}$ & $\begin{array}{l}\text { Complication } \\
(n=33)\end{array}$ & $\begin{array}{l}\text { Recovery } \\
(n=120)\end{array}$ \\
\hline$C G(n=20)$ & $\begin{array}{l}0.347 \\
(0.299-0.430)\end{array}$ & & & & & \\
\hline$\overline{\mathrm{AP}(n=169)}$ & & $\begin{array}{l}0.600 \\
(0.375-0.840)(* *)\end{array}$ & $\begin{array}{l}0.451 \\
(0.286-0.597)(*)(a)\end{array}$ & $\begin{array}{l}0.343 \\
(0.212-0.577)(a),(b),(c)\end{array}$ & $\begin{array}{l}0.473 \\
(0.230-0.750)\end{array}$ & $\begin{array}{l}0.359 \\
(0.242-0.523)(a)\end{array}$ \\
\hline
\end{tabular}

Values expressed as median (IQR).

$\left(^{*}\right) p<0.05$ with respect to $C G ;\left(^{* *}\right) p<0.001$ with respect to a CG (Mann-Withney test).

(a) $p<0.05$ with respect to $24 \mathrm{~h}$; (b) $p<0.05$ with respect to $48 \mathrm{~h}$; (c) $p<0.05$ with respect to a complication (Wilcoxon test for paired data).

tance. At 96 hours and at recovery, no statistical difference was found between AP and CG. When complications were detected, MDAc in AP was higher than in $\mathrm{CG}$, but the difference did not reach statistical significance (Table I).

MDAc progressively decreased from 24 to 96 hours, with levels at each time point being significantly lower than at the previous one. At recovery, MDAc was significantly lower than at 24 hours. The development of complications was associated with a rise of MDAc to levels similar to those at 24 and 48 hours (Table I).

\section{MDAc according to severity}

At 24 hours MDAc was higher in patients with either SAP or MAP compared to CG. At 48 hours patients with SAP but not those with MAP, showed significantly higher levels than CG. At 96 hours and at recovery, MDAc was similar in the three groups (Table II).

The evolution of MDAc over time was different in MAP and SAP. On the one hand, patients with MAP showed a significant decrease in MDAc at 48 hours to levels similar to those at recovery, and on the other hand, in patients with SAP the decrease was not significant until 96 hours from admittance (Table II, Fig. 1).

\section{Relationship between MDAc and analytical parameters}

Table III shows the analytical parameters at 24 and 48 hours in MAP and SAP. The relationship between MDAc and analytical parameters was poor.

\section{MDAc as prognostic factor of severity}

ROC curves of MDAc at 24 and 48 hours were drawn. The area under the curve of these parameters was no significant. Consequently, as no cut-off value could be identified, those parameters were not included in the logistic regression model. The multivariate analysis showed that the only risk factor of severity was the presence of three or more Ranson criteria -odds ratio $12.2,95 \%$ confidence interval (2.7-54.7), $\mathrm{p}=0.001-$.

\section{DISCUSSION}

Severe AP can be considered a multisystemic disease, with a course that is determined by the development of a SIRS. One of the most interesting aspects in the pathogenesis and early evolution of AP is the role played by OS,

Table II. MDAC $(\mu \mathrm{M})$ at each time point and evolution in MAP and SAP

\begin{tabular}{|c|c|c|c|c|c|c|}
\hline & & $\begin{array}{l}24 \text { hours } \\
(n=169)\end{array}$ & $\begin{array}{l}48 \text { hours } \\
(n=169)\end{array}$ & $\begin{array}{l}96 \text { hours } \\
(n=169)\end{array}$ & $\begin{array}{l}\text { Complication } \\
(n=33)\end{array}$ & $\begin{array}{l}\text { Recovery } \\
(M A P=99 ; S A P=21)\end{array}$ \\
\hline$C G(n=20)$ & $\begin{array}{l}0.347 \\
(0.299-0.430)\end{array}$ & & & & & \\
\hline $\mathrm{MAP}(\mathrm{n}=136)$ & & $\begin{array}{l}0.583 \\
(0.395-0.821)^{*}\end{array}$ & $\begin{array}{l}0.440 \\
(0.281-0.589)(a)\end{array}$ & $\begin{array}{l}0.355 \\
(0.212-0.580)(a)\end{array}$ & & $\begin{array}{l}0.360 \\
(0.240-0.542)(a)\end{array}$ \\
\hline$\overline{\mathrm{SAP}}(\mathrm{n}=33)$ & & $\begin{array}{l}0.615 \\
(0.356-0.972)^{*}\end{array}$ & $\begin{array}{l}0.514 \\
(0.347-0.707)^{*}\end{array}$ & $\begin{array}{l}0.328 \\
(0.210-0.518)(a),(b),(c)\end{array}$ & $\begin{array}{l}0.473 \\
(0.230-0.750)\end{array}$ & $\begin{array}{l}0.359 \\
(0.278-0.489)\end{array}$ \\
\hline
\end{tabular}

Values expressed as median (IQR).

$\left.{ }^{*}\right) p<0.05$ with respect to CG (Mann-Withney test).

(a) $p<0.05$ with respect to 24 h; (b) $p<0.05$ with respect to $48 \mathrm{~h}$; (c) p $<0.05$ with respect to Complication (Wilcoxon test for paired data). 


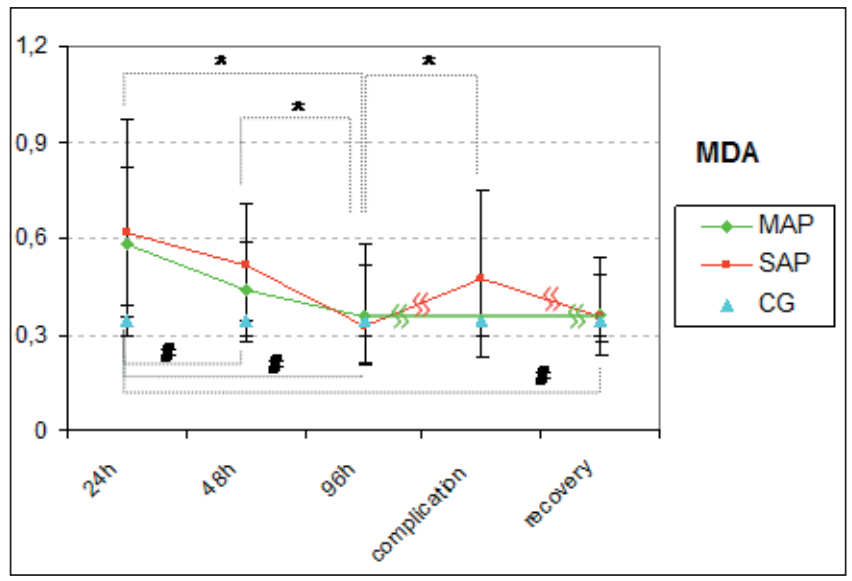

Fig. 1. Evolution of MDAc over time in MAP and SAP. $\left(^{*}\right): p<0.05$, in SAP, Wilcoxon test. (\#): $p<0.05$, in MAP, Wilcoxon test.

which has potential to activate and perpetuate the inflammatory cascade $(2,3,24,25)$. Although there is considerable amount of evidence showing enhanced OS in patients with AP, to our knowledge, only five studies have thoroughly evaluated OS in the early phase of AP $(7,8,12,14,15)$, so we decided to carry out investigation to better understand the dynamics of OS in this early phase and its relationship with the severity of the disease in a homogeneous group of patients with respect to the time of evolution of symptoms and the absence of diseases related to OS, apart from the AP.
Our study group was formed by 169 patients, one of the largest groups evaluated to date together with the group of Rahman et al. (13). The cause of the episode was not established in $18.9 \%$ of cases, which is similar to the figures given in the latest British guidelines (26). One could think that our series shows a low-severity group, as the need of intensive management in the Intensive Care Unit was low, but this could be explained by the fact that in our Department patients can be monitored as in a high-dependence unit. In fact, patients with SAP account for $19.5 \%$, similar to the percentages found in consensus papers $(1,27)$. In contrast, some authors have reported a higher percentage of patients with SAP (from 33.8 to $50.8 \%$ ) $(7,8,10,12,21)$, and others evaluated exclusively patients with SAP or severe-predicted pancreatitis (9), which could overestimate the role of OS in AP. Taking into account these aspects, we can consider our series as representative of daily clinical practice, avoiding selection biases.

In patients with AP we found high MDAc at 24 and 48 hours, while at 96 hours from admittance the levels were within the normal range. MDAc peaked at 24 hours and decreased progressively to normal values at 96 hours. When any complication was detected, it was observed that MDAc rose to levels similar to those at 24 and 48 hours. This evolution in MDAc is in accordance with that described by Abu-Hilal et al., who found a progressive decrease in MDA levels from 12 to 96 hours, when they were similar to the CG (21). In contrast with this description, other authors have reported a different evolution. Wereszczynska-Seimiatkowska et al. did not find any decrease in MDA until the

Table III. Analytical parameters at $\mathbf{2 4}$ and $\mathbf{4 8}$ hours

\begin{tabular}{|c|c|c|c|c|}
\hline \multirow[t]{2}{*}{ Parameter } & \multicolumn{2}{|c|}{24 hours } & \multicolumn{2}{|c|}{48 hours } \\
\hline & $M A P(n=136)$ & $\operatorname{SAP}(n=33)$ & $M A P(n=136)$ & $\operatorname{SAP}(n=33)$ \\
\hline Leucocytes (x106/l) & $10,110(9,380-10,850)$ & $13,810(11,680-15,930)^{*}$ & $9,830(9,020-10,630)$ & $12,880(10,990-14,780)^{*}$ \\
\hline Neutrophiles (x10/l) & $8,210(7,460-8,970)$ & $12,220(10,230-14,210)^{*}$ & $7,730(9,710-8,540)$ & $11,060(9,260-12,860)^{*}$ \\
\hline Hemoglobine (g/dl) & $12.7(12.4-12.9)$ & $13.6(12.8-14.4)^{*}$ & $12.7(12.4-12.9)$ & $12.5(11.8-13.2)$ \\
\hline Hematocrit (\%) & $37.5(36.8-38.2)$ & $40.8(38.1-43.4)^{*}$ & $37.5(36.8-38.2)$ & $37.0(35.0-39.1)$ \\
\hline Glucemia (mg/dl) & $114(107-120)$ & $167(143-191)^{\star}$ & $102(97-107)$ & $140(118-161)^{*}$ \\
\hline Urea (mg/dl) & 30.5 (27.9-33.1) & $49.1(38.8-59.4)^{*}$ & $24.0(21.6-26.4)$ & $45.0(32.7-57.3)^{*}$ \\
\hline Creatinine (mg/dl) & $0.87(0.83-0.90)$ & $1.17(1.00-1.33)^{*}$ & $0.86(0.82-0.89)$ & $1.20(0.96-1.45)^{*}$ \\
\hline AST (U/l) & $145(108-183)$ & $122(78-167)$ & $60(48-72)$ & $56(39-72)$ \\
\hline $\mathrm{LDH}(\mathrm{U} / \mathrm{l})$ & $461(406-517)$ & $571(505-636)$ & $363(331-394)$ & $627(491-763)^{\star}$ \\
\hline Calcium (mg/dl) & $8.46(8.33-8.59)$ & $8.29(7.98-8.60)$ & $8.51(8.38-8.64)$ & $8.23(7.81-8.66)$ \\
\hline Albumin (g/dl) & $3.55(3.46-3.64)$ & $3.61(3.09-4.14)$ & $3.47(3.37-3.57)$ & $3.21(2.93-3.50)$ \\
\hline Base Excess (mEq/l) & $-0.27(-1.02$ a 0.47$)$ & $-2.51(-4.44 ;-0.58)^{*}$ & & \\
\hline $\mathrm{pO}_{2}(\mathrm{mmHg})$ & $88.9(80.5-97.2)$ & $80.7(65.6-95.7)$ & & \\
\hline Fibrinogen (g/l) & $4.76(4.42-5.11)$ & $5.57(4.35-6.79)$ & $7.00(5.22-8.77)$ & $8.21(6.93-9.49)$ \\
\hline ESR (mm/1 hour) & $27.1(3.6-50.7)$ & $27.8(13.6-37.9)$ & $30.2(25.5-34.8)$ & $54.8(35.1-74.4)^{\star}$ \\
\hline $\mathrm{CRP}(\mathrm{mg} / \mathrm{l})$ & $45.5(35.5-55.5)$ & $122.3(82.6-162.1)^{*}$ & $108.5(90.7-126.3)$ & $237.0(187.1-286.9)^{*}$ \\
\hline $\operatorname{MDAc}(\mu \mathrm{M})$ & $0.583(0.395-0.821)$ & $0.615(0.356-0.972)$ & $0.440(0.281-0.589)$ & $0.514(0.347-0.707)$ \\
\hline
\end{tabular}

Values expressed as mean (C195\%), MDAc expressed as mediam (IQR).

Statistical analysis: Student-t test; MDAc compared with Mann-Whitney test. $\left.{ }^{*}\right)$ : $p<0.05$, with respect to MAP 
fifth day from admittance, and levels at $10^{\text {th }}$ day remained higher than the CG (8). Tsai et al. described a decrease in MDAc at $7^{\text {th }}$ and $14^{\text {th }}$ day with respect to that of admittance, but it did not reach the normal range (7). Winterbourn et al. did not find any difference between AP and controls at any time point, nor any variation over time (11). Dziurkowska-Marek et al. appreciated an early increase in MDA levels to the fifth day, followed by a decrease that did not reach the normal values (14). Thareja et al. found a decrease in TBARS from the first to the seventh day, where it did not reach CG levels (15). The different evolutive pattern in OS could be explained, in part, by the method used to assess lipid peroxidation. Tsai et al. and Dziurkowska-Marek et al. determined TBARS, less specific than MDA measurement by HPLC (28); the method chosen by Wereszczynska-Seimiatkowska et al. to determine MDA was not based on HPLC (29), so it could be less specific; Wintherbourn et al. measured plasmatic MDA bound to proteins (30), while we decided to determine free serum MDA (23).

It is assumed that patients with MAP show a lower degree and a better evolution of OS than patients with SAP; however, the studies on this subject that analyze MDA or TBARS are controversial. On the one hand, when lipid peroxidation is assessed by means of TBARS determination or by a low-specific MDA determination, differences between MAP and SAP are found both in the degree and evolution of lipid peroxidation $(7,8,10,14,19)$. On the other hand, authors that determine MDAc by HPLC do not find differences in its degree or evolution $(11,17)$. Similarly to the latter, in our study only indirect data suggesting a higher degree of lipid peroxidation in SAP were found. Firstly, these patients presented higher MDAc than CG at 48 hours, while patients with MAP did not. The decrease in MDAc was not significant until 96 hours from admittance in patients with SAP, whereas in MAP this was evident at 48 hours. Finally, the detection of complications was associated with MDAc similar to the levels at 24 and 48 hours.

One of the most interesting aspects in AP is the prediction of severity. The accuracy of the current severity criteria is not optimal, and it has been described that the corelation between clinical-analytical criteria and radiologic severity is low (31). Some authors have proposed MDAc as a predictive parameter of severity $(8,10)$. However, the studies that have included it in a multivariate analysis (17), as we did, have not found MDAc to be a good prognostic factor of severity.

Oxygen radical species are known to be important in intracellular, intercellular and interorgan signalling for the initiation and development of pancreatitis-associated MOF, by activating nuclear factor transcription to produce a number of cytokines, stimulating the expression of adhesion molecules on leukocytes and endothelial cell surfaces and initiating inflammatory reactions in extrapancreatic organs or tissues (25). In AP, mortality is closely related to the development of persistent MOF (32-35). In the present study we have found that the main difference between MAP and SAP, with regard to the presence of OS, is not its magnitude but its persistence during the first 48 hours from admittance (in our patient population, this represents 72 hours from the onset of abdominal pain). This approach is in accordance with the role played by OS in the pathophysiology of SIRS and MOF, and, moreover, with the close relationship between persistent MOF and severity.

An attractive hypothesis drawn from this observation is the possible "window period" in which a treatment addressed to control OS could lead to a more favourable outcome of AP. Recently, Siriwardena et al. (36) reported a nicely designed randomized, double-blinded, placebo-controlled trial on intravenous antioxidant treatment for SAP. The study was performed on patients with predicted SAP, managed according to the United Kingdom guidelines for the management of AP (26), and the authors found that antioxidant supplementation was not useful to avoid MOF, ameliorate patient outcomes or reduce length of hospital stay. However, one of the inclusion criteria was that "patients should be enrolled within 72 hours of admission to hospital", a criterion which deserves further comments. The authors do not describe the time from the onset of abdominal pain (accepted as the onset of pancreatitis) to hospital admittance, nor do they establish this parameter as an inclusion criterion; thus, one could suppose that patients with an advanced disease were included in the trial. It is known that OS is an early event in the course of AP (in our study patients with SAP showed high MDAc only during the first 48 hours from admittance, a maximum of 72 hours from the onset of pain), and including patients with long evolution symptoms could underestimate an effect of antioxidant treatment.

Learning about pathophysiological mechanisms taking part in the development of AP in humans can help us to better understand the evolution of the disease and to improve its management. As previously described, we found an early OS in patients with AP, shown by the high level of MDAc. With respect to the severity of the disease, in our study, the main difference between MAP and SAP is the persistent OS in the latter. Although current evidence does not support the use of antioxidant therapy in patients with AP, our findings suggest a short "therapeutic window", limited to the 72 hours from the onset of symptoms, which deserves further investigation.

\section{ACKNOWLEDGEMENTS}

This study has been partly funded by a grant from the Instituto de Salud Carlos III (CO312) and by other grant from the Universitat de Valencia. (V Segles grant).

We acknowledge Ms Miriam Phillips her help in preparing the English version of the manuscript.

\section{REFERENCES}

1. Bradley EL III. A clinically based classification system for acute pancreatitis. Arch Surg 1993;128:586-90.

2. Braganza JM. Towards a novel treatment strategy for acute pancreatitis. Reappraisal of the evidence on aetiogenesis. Digestion 2001;63:69-91. 
3. Norman JG. New approaches to acute pancreatitis: Role of inflammatory mediators. Digestion 1999; 60 (suppl 1):57-60.

4. Sanfey H, Bulkley GB, Cameron JL. The role of oxygen free radicals in the pathogenesis of acute pancreatitis. Ann Surg 1984;200:405-12.

5. Scott P, Bruce C, Schofield D, Shiel N, Braganza JM, McCloy RF. Vitamin C status in patients with acute pancreatitis. Br J Surg 1993; 80: 750-4.

6. Braganza JM, Scott P, Bilton D, Schofield D, Chaloner C, Shiel N, et al. Evidence of early oxidative stress in acute pancreatitis. Clues for correction. Int J Pancreatol 1995;17:69-81.

7. Tsai K, Wang SS, Chen TS, Kong CW, Chang FY, Lee SD, et al. Oxidative stress: an important phenomenon with pathogenic significance in the progression of acute pancreatitis. Gut 1998;42:850-5.

8. Wereszczynska-Seimiatkowska U, Dabrowski, A, Jedynak M, Gabryelewicz A. Oxidative Stress as an early prognostic factor in acute pancreatitis (AP): its correlation with serum phospholipase A2 (PLA2) and plasma polymorphonuclear elastase (PMN-E) in different-severity forms of human AP. Pancreas 1998;17:163-8.

9. Curran FJ, Sattar N, Talwar D, Baxter JN, Imrie CW. Relationship of carotenoid and vitamins $\mathrm{A}$ and $\mathrm{E}$ with the acute inflammatory response in acute pancreatitis. Br J Surg 2000;87:301-5.

10. Park BK, Chung JB, Lee JH, Suh JH, Park SW, Song SY, et al. Role of free radicals in patients with acute pancreatitis. World J Gastroenterol 2003;9:2266-9.

11. Winterbourn CC, Bonjam MJ, Buss J, Abu-Zidan FM, Windsor JA. Elevated protein carbonyls as plasma markers of oxidative stress in acute pancreatitis. Pancreatology 2003;3:375-82.

12. Wereszczynska-Seimiatkowska U, Mroczko D, Siemiatkowski A, Szmitkowski M, Borawska M, Kosel J. The importance of interleukin 18 , glutathione peroxidase, and selenium concentration changes in acute pancreatitis. Dig Dis Sci 2004;49:642-50.

13. Rahman SH, Ibrahim K, Larvin M, Kingsnorth A, McMahon MJ. Association of antioxidant enzyme gene polymorphisms and glutathione status with severe acute pancreatitis. Gastroenterology 2004;126:1312-22.

14. Dziurkowska-Marek A, Marek TA, Nowak A, Kacperek-Hartleb T, Wierka D, Nowakowska-Dulawa E. The dynamics of oxidant/antioxidant balance in the early phase of human acute biliary pancreatitis. Pancreatology 2004;4:215-22.

15. Thareja S, Bhardwaj P, Sateesh J, Saraya A. Variations in the levels of oxidative stress and antioxidants during early acute pancreatitis. Trop Gastroenterol 2009;30:26-31.

16. Bonham MJ, Abu-Zidan FM, Simovic MO, Sluis KB, Wilkinson A, Winterbourn CC, et al. Early ascorbic acid depletion is related to the severity of acute pancreatitis. Br J Surg 1999;86:1296-301.

17. Abu-Zidan FM, Bonham MJ, Windsor JA. Severity of acute pancreatitis: a multivariate analysis of oxidative stress markers and modified Glasgow criteria. Br J Surg 2000;87:1019-23.

18. Halliwell B. Oxidative stress markers in human disease: application to diabetes and to evaluation of the effects of antioxidants. In: Antioxidants in Diabetes Management. Packer L, Rosen P, Tritschler HJ, King GL, Azzi A, eds. Marcel Dekker INC. New York 2000:33-52.

19. Du WD, Yuan ZR, Sun J, Tang JX, Cheng AQ, Shen DM, et al. Therapeutic efficacy of high-dose vitamin $\mathrm{C}$ on acute pancreatitis and its potential mechanisms. World J Gastroenterol 2003;9:2565-9.
20. Sajewicz W, Milnerowicz S, Nabzdyk S. Blood plasma antioxidant defense in patients with pancreatitis. Pancreas 2006;32:139-44.

21. Abu-Hilal M, McPhail MJW, Marchand L, Johnson CD. Malondialdehyde and superoxide dismutase as potential markers of severity in acute pancreatitis. JOP 2006;7:185-92.

22. Richard MJ, Guiraud P, Meo J, Favier A. High performance liquid chromatography separation of malondialdehyde thiobarbituric acid adduct in biological materials (plasma and human cell) using a commercially available reagent. J. Chromatogr 1992;577:9-18.

23. Romero MJ, Bosch-Morell F, Romero B, Rodrigo JM, Serra MA, Romero FJ. Serum malondialdehyde: possible use for the clinical management of chronic hepatitis C patients. Free Radic Biol Med 1998; 25:993-7.

24. Norman J. The role of cytokines in the pathogenesis of acute pancreatitis. Am J Surg 1998;175:76-83.

25. Shi C, Andersson R, Wang X. Potential role of reactive oxygen species in pancreatitis-associated multiple organ dysfunction. Pancreatology 2005;5:492-500.

26. United Kingdom guidelines for the management of acute pancreatitis. UK Working Party on Acute Pancreatitis. Gut 2005;54 (suppl 3):iii1iii9.

27. United Kingdom guidelines for the management of acute pancreatitis. Gut 1998; 42(suppl 2):S1-S13.

28. Janero DR. Malondialdehyde and thiobarbituric acid-reactivity as diagnostic indices of lipid peroxidation and peroxidative tissue injury. Free Radical Biol Med 1990;9:515-40.

29. Salaris SC, Babbs CF. A rapid, widely applicable screen for drugs that suppress free radical formation in ischemia/reperfusion. J Pharmacol Methods 1988;20:335-45.

30. Young IS, Trimble ER. Measurement of malondialdehyde in plasma by high performance liquid chromatography with fluorimetric detection. Ann Clin Biochem 1991;28:504-8.

31. Lujano-Nicolás LA, Pérez-Hernández JL, Durán-Pérez EG, SerraldeZúñiga AE. Correlation among clinical, biochemical and tomographic criteria in order to evaluate the severity in acute pancreatitis. Rev Esp Enferm Dig 2010;102:376-80

32. Buter A, Imrie CW, Carter CR, Evans S, Mckay CJ. Dynamic nature of early organ dysfunction determines outcome in acute pancreatitis. Br J Surg 2002;89:298-302.

33. Johnson CD, Abu-Hilal M. Persistent organ failure during the first week as a marker of fatal outcome in acute pancreatitis. Gut 2004;53:13404.

34. Halonen KI, Pettila V, Leppaniemi AK, Kemppainen EA, Puolakkainen PA, Haapiainen RK. Multiple organ dysfunction associated with severe acute pancreatitis. Crit Care Med 2002;30:1274-79.

35. Mofidi R, Duff MD, Wigmore SJ, Madhavan KK, Garden OF, Parks RW. Association between early systemic inflammatory response, severity of multiorgan dysfunction and death in acute pancreatitis. Br J Surg 2006;93:738-44.

36. Siriwardena AK, Mason JM, Balachandra S, Bagul A, Galloway S, Formela L, et al. Randomised, double blind, placebo controlled trial of intravenous antioxidant (n-acetylcysteine, selenium, vitamin C) therapy in severe acute pancreatitis. Gut 2007;56:1439-44. 